\title{
BIG RIP AND OTHER SINGULARITIES IN ISOTROPIC HOMOGENEOUS COSMOLOGICAL MODELS WITH ARBITRARY EQUATION OF STATE
}

\author{
S.L. Parnovsky \\ Astronomical Observatory, Taras Shevchenko National University of Kyiv, \\ 3 Observatorna Str., 04053, Kyiv, Ukraine, par@observ.univ.kiev.ua
}

\begin{abstract}
We study the possible types of future singularities in the isotropic homogeneous cosmological models for the arbitrary equation of state of the contents of the Universe. We obtain all known types of these singularities as well as two new types using a simple approach. No additional singularity types are possible. We name the new singularities type "Big Squeeze" and "Little Freeze". The "Big Squeeze" is possible only in the flat Universe after a finite time interval. The density of the matter and dark energy tends to zero and its pressure to minus infinity. This requires the dark energy with a specific equation of state that has the same asymptotical behaviour at low densities as the generalised Chaplygin gas. The "Little Freeze" involves an eternal expansion of the Universe. Some solutions can mimic the $\Lambda$ CDM model.
\end{abstract}

Keywords: Relativistic cosmology

\section{Introduction}

During almost a century, cosmologists considered only two possible scenarios of the future of our Universe - an eternal expansion of open or flat Universe or future recollapse of the closed Universe with the "Big Crunch". Nowadays we know that the Universe contains not only several types of matter, including the dark matter, baryonic matter and massless particles, but also the mysterious dark energy (DE). We know about its existence only for the last few decades. Honestly, we know very little about DE properties, in particular about the DE equation of state.

Even for the simplest type of the DE equation of state

$$
p=w \rho
$$

with $w=$ const, where $p$ is the pressure and $\rho$ is the mass density, the Universe can meet its end in absolutely different way. If $w<-1$ we deal with so-called phantom energy. In this case during the finite time period the matter and energy density, the Hubble parameter $H$ and the scale factor of the Universe $a$ increase to infinity. Such type of possible future singularity was discovered by Caldwell, Kamionkowski and Weinberg (2003) and called "Big Rip".

Note that the latest estimations of the $w$ value do not reject this possibility. The data on the cosmic microwave background spectra from the Planck and WMAP satellites together with ground measurements and data from baryonic acoustic oscillations (BAO) provide the estimation $w=-1.13_{-0.25}^{+0.23}$ at $95 \%$ confidence level (CL). The 9-year data from the WMAP satellite plus the determination of the Hubble constant and BAO data provide estimations $w=-1.073_{-0.089}^{+0.090}$ for the flat Universe and $w=-1.19 \pm 0.12$ for the non-flat Universe at $68 \%$ CL. Adding 472 type Ia supernovae data improves these estimations to $w=$ $-1.084 \pm 0.063$ and $w=-1.122_{-0.067}^{+0.068}$, respectively.

Thus, the possibility of the "Big Rip" sealing the fate of the Universe is not to be taken lightly. This is not the only theoretically possible type of cosmological singularity except "Big Bang" and "Big Crunch". Their first classification was carried out by Nojiri, Odintsov and Tsujikawa (2005). Four possible types were found for the singularities at $t=t_{0}$ with finite $t_{0}$. They include:

- Type I $a, \rho,|p| \rightarrow \infty$ ("Big Rip")

- Type II $a \rightarrow a_{0} ; \rho \rightarrow \rho_{0} ;|p| \rightarrow \infty$ ("sudden")

- Type III $a \rightarrow a_{0} ; \rho,|p| \rightarrow \infty$ (it was named "Big Freeze" lately)

- Type IV $a \rightarrow a_{0} ; \rho,|p| \rightarrow 0$ and higher derivatives of the Hubble parameter $H$ diverge.

There are some singularities with $t_{0}=\infty$, too. The "Little Rip" singularity (Frampton, Ludwick and Scherrer, 2012) similar to the "Big Rip", but with eternal expansion is among them.

Some types of singularities were found and demonstrated for some specific equations of state. Cosmologists considered the particular cases of the phantom generalised Chaplygin gas equation of state, tachyon field, scalar fields with specific potentials, etc. Naturally, a question arose, whether all the possible singularity types have been considered. 
In this article we try to give an exhaustive answer to this question for the isotropic and homogeneous Universe. To make it worse, in addition to unknown DE equation of state we have three possible signs of space curvature. We are interesting in the complete list of the possible types of future singularities for an arbitrary equation of state for three signs of space curvature. We consider an arbitrary equation of state $p(\rho)$ without any constrains except $\rho \geq 0$. In particular we do not use the strong energy condition $\rho+3 p>0$.

\section{The search for future singularities in FLRW Universe}

We consider the homogeneous isotropic Universe with the Friedmann-Lemaître-Robertson-Walker (FLRW) metric

$$
d s^{2}=d t^{2}-a(t)^{2}\left[d \chi^{2}+F^{2}(\chi) d O^{2}\right],
$$

where $a(t)$ is the scale factor, $d O^{2}=d \Theta^{2}+\cos ^{2}(\Theta) d \varphi^{2}$ is the distance element on a unit sphere, $F(\chi)=\sin (\chi)$ and $k=1$ for the closed Universe, $F(\chi)=\sinh (\chi)$ and $k=-1$ for the open one, and $F(\chi)=\chi$ and $k=0$ for the spatially flat models. We use the system of units in which $G=1$ and $c=1$. This Universe is filled by all kinds of matter and dark energy with a mass density $\rho$ and an effective pressure $p(\rho)$. In this system of units the energy density $\varepsilon$ coincides with $\rho$. The Einstein equations for the metric (2) reduce to the well-known Friedmann equations. We need the expression for the Hubble parameter $H=a^{-1} d a / d t$

$$
H^{2}=\frac{8 \pi}{3} \rho-\frac{k}{a^{2}}
$$

and the hydrodynamical equation or the energy conservation equation

$$
\frac{d \rho}{d t}=-3(\rho+p) H
$$

The Friedmann equation for the scale factor

$$
\frac{d^{2} a}{d t^{2}}=-\frac{4 \pi}{3} a(\rho+3 p)
$$

follows from the equations (3) and (4).

\subsection{Flat model}

We start from the flat model with $k=0$. The equation (3) provides the expression $H=(8 \pi \rho / 3)^{1 / 2}$. After substituting it into (4) we obtain a simple equation with the solution

$$
\Delta t=t_{0}-t_{1}=-\frac{1}{2(6 \pi)^{1 / 2}} \int_{\rho_{1}}^{\rho_{0}} \frac{d \rho}{\rho^{1 / 2}(\rho+p(\rho))} .
$$

Here the subscript 1 corresponds to the initial parameters (i.e. $t_{1}$ is "now") and the subscript 0 corresponds to the parameters of the Universe in the future at time $t_{0}$ after a time interval $\Delta t$. We will denote the instant of time of any terminal cosmological singularity as $t_{0}$, and use (6) to analyse their properties. After finding the dependence $\Delta t(\rho)$ we find the inverse function $\rho(\Delta t)$ and $H(\Delta t)$, the integration of the last one gives $\ln (a)$.

The first thing to check is the finiteness of $\Delta t$. If the integral in (6) diverges we obtain $t_{0}=\infty$ and this case deals with the asymptotic evolution in the future. An example of such solution is the "Little Rip".

We are going to go over all possible types of singularity. We consider three possible cases for $\rho_{0}$. It can be infinite, finite and nonzero, or equal to zero. Let us consider it one by one.

\subsubsection{Infinite terminal density}

Let us start with a well-known "Big Rip" singularity to illustrate our approach. We consider the equation of state (1). If $w=-1$ we deal with the effective cosmological constant. According to (4) in this case the density and the pressure are constant. If $w>-1$ the values of $\rho$ and $H$ decrease in time because of (4). If $w<-1$ the values of $\rho$ and $H$ increase due to (4) and become infinite at time $t_{0}$. Equation (6) gives us in this case the relations

$$
\rho_{1}=\frac{1}{6 \pi(1+w)^{2} \Delta t^{2}}, H=\frac{2}{3|1+w| \Delta t} .
$$

This is the so-called "Big Rip" case. The scale factor of the Universe diverges $a \propto \Delta t^{-\frac{2}{3|1+w|}}$.

A somewhat similar case is when $w$ is not constant, but asymptotically tends to $-1: \rho / p \underset{\rho \rightarrow \infty}{\longrightarrow}-1$. Let us assume that it follows the power law

$$
\rho+p \underset{\rho \rightarrow \infty}{\longrightarrow}-A \rho^{\alpha}
$$

with $\alpha<1, A=$ const. The integral in (6) is finite at $1 / 2<\alpha<1$. In this case we have the "Big Rip" with $H \propto \Delta t^{1 /(1-2 \alpha)}, \ln a \propto \Delta t^{2(1-\alpha) /(1-2 \alpha)}$. It occurs later and has a sharper shape for the same initial value $\rho_{1}$ in comparison with the equation of state $(1)$.

If $\alpha<1 / 2$ the integral in (6) becomes divergent and we have to put $t_{0}=\infty$. This is the so-called "Little Rip" introduced in (Frampton, Ludwick and Scherrer, 2012). In this case we rewrite (6) in the form

$$
\Delta t=t-t_{1}=-\frac{1}{2(6 \pi)^{1 / 2}} \int_{\rho_{1}}^{\rho(t)} \frac{d \rho}{\rho^{1 / 2}(\rho+p(\rho))} .
$$

This case corresponds to an eternally accelerating expansion of the Universe: $H \propto t^{1 /(1-2 \alpha)}, \ln a \propto$ $t^{2(1-\alpha) /(1-2 \alpha)}$.

In the intermediate case $\alpha=1 / 2$ we must take into account a possible logarithmic divergence and consider 
the equation of state with the asymptote $\rho+p \underset{\rho \rightarrow \infty}{\longrightarrow}$ $-A \rho^{1 / 2}(\ln \rho)^{\beta}$. At $\beta>1$ we deal with the unconventional "Big Rip" with $\ln \rho \propto \Delta t^{1 /(1-\beta)}$, at $\beta<1$ we deal with the "Little Rip" with $\ln \rho \propto t^{1 /(1-\beta)}$. At $\beta=1$ we consider the equation of state with the asymptotic $\rho+p \underset{\rho \rightarrow \infty}{\longrightarrow}-A \rho^{1 / 2}(\ln \rho)(\ln \ln \rho)^{\gamma}$. There is the "Big Rip" with $\ln \ln \rho \propto \Delta t^{1 /(1-\gamma)}$ at $\gamma<1$ and the "Little Rip" with $\ln \ln \rho \propto t^{1 /(1-\gamma)}$ at $\gamma>1$.

So far we considered cases with $a \underset{\rho \rightarrow \infty}{\longrightarrow} \infty$, but this is not required. For example, a type III singularity has finite $t_{0}$ and $a_{0}$ values, but $\rho, H,|p| \underset{t \rightarrow t_{0}}{\longrightarrow} \infty$. Let us consider this type of singularity. From $H=$ $a^{-1} d a / d t \underset{t \rightarrow t_{0}}{\longrightarrow} \infty$ and $a(t) \underset{t \rightarrow t_{0}}{\longrightarrow} a_{0}$ we see that $a(t)$ is regular, but $d a / d t$ diverges at $t=t_{0}$. This is possible if the scale factor has a power-law asymptote

$$
a(t) \underset{t \rightarrow t_{0}}{\longrightarrow} a_{0}-B\left(t_{0}-t\right)^{\lambda}
$$

with $0<\lambda<1$. This yilds $H \underset{t \rightarrow t_{0}}{\longrightarrow} \lambda B\left(t_{0}-t\right)^{\lambda-1} / a_{0}$. From (3) we obtain for this case $\rho(t) \propto\left(t_{0}-t\right)^{2(\lambda-1)}$. After substituting these expressions in (4) we get $\rho(t)+$ $p(t) \propto\left(t_{0}-t\right)^{\lambda-2}$. This corresponds to the equation of state (8) with $\alpha=\frac{2-\lambda}{2-2 \lambda}, \lambda=\frac{2 \alpha-2}{2 \alpha-1}$. In this case $\alpha>1$ and $|p| \propto \rho^{\alpha} \gg \rho$ in the vicinity of the singularity.

Let us consider this type of singularity directly from (6). If $\rho \underset{t \rightarrow t_{0}}{\longrightarrow} \infty$ but $\rho / p \underset{\rho \rightarrow \infty}{\longrightarrow} 0$, e.g. $p(\rho) \underset{\rho \rightarrow \infty}{\longrightarrow}$ $-A \rho^{\alpha}$ with $\alpha>1, A=$ const we also have a singularity with $H \propto \Delta t^{1 /(1-2 \alpha)}, \ln a \propto \Delta t^{2(1-\alpha) /(1-2 \alpha)}=\Delta t^{\lambda}$. Note that at $\alpha<1$ we get the "Big Rip" case considered above. But in the case of the "Big Freeze" singularity the scale factor tends to some constant value.

If we deal with the power law (10) for the scale factor with some noninteger $\lambda>1$ we have no "Big Freeze" singularity, but some higher derivatives of $H$ diverge. If $1<\lambda<2$ both parts of the Friedmann equation (5) diverge, if $\lambda>2$ both of them tend to zero. This case corresponds to $\rho \underset{t \rightarrow t_{0}}{\longrightarrow} 0,|p| \underset{t \rightarrow t_{0}}{\longrightarrow} \infty$ and we will consider it later.

Is a version of the "Big Freeze" with $t_{0}=\infty$ possible? It could be named the "Little Freeze" similarly to the situation with the "Big Rip" and the "Little Rip". In this case instead of (10) we consider an asymptotic behaviour of the scale factor in the form $a(t) \underset{t \rightarrow \infty}{\longrightarrow} a_{0}-B t^{\lambda}$ with $\lambda<0$. According to $(3)$ and (4) we have in this case $\rho(t) \propto t^{2 \lambda-2} \underset{t \rightarrow \infty}{\longrightarrow} 0$ and $p(t) \propto t^{\lambda-2} \underset{t \rightarrow \infty}{\longrightarrow} 0$. This possibility will be considered later, too.

\subsubsection{Finite terminal density}

Let us consider singularities with a nonsingular $\rho \underset{t \rightarrow t_{0}}{\longrightarrow} \rho_{0} \neq 0$. In this case all nontrivial solutions require $p+\rho$ factor to diverge or vanish according to
(6). In the first case $|p| \rightarrow \infty$, the second one corresponds to the crossing the line $\rho+p=0$. It corresponds to the equation of state of the cosmological constant, separating the phantom energy domain with an effective $w<-1$ from the domain of not so exotic matter $w>-1$. We will see that the possibility of such crossing depends on the parameters of the equation of state.

We start with considering solutions with finite $t_{0}$. Both cases could be described by a single power-law asymptote of the equation of state

$$
\rho+p(\rho) \underset{\rho \rightarrow \rho_{0}}{\longrightarrow} C\left(\rho-\rho_{0}\right)^{\mu}
$$

with $C=$ const.

At $\mu<0$ the modulus of the pressure tends to infinity, at $\mu>0$ the $\rho+p$ reaches zero. The finiteness of $t_{0}$ is possible only at $\mu<1$. In this case we have $\rho(t)-\rho_{0} \propto \Delta t^{1 /(1-\mu)}, \rho(t)+p(t) \propto \Delta t^{\mu /(1-\mu)}$. The singularity with $\mu<0$ and $|p| \underset{\rho \rightarrow \rho_{0}}{\longrightarrow} \infty$ is referred to as the type II or sudden singularity. The value of $H$ tends to finite $H_{0}$, so the scale factor linearly increases.

The achievement of $\rho+p=0$ condition in finite time is possible if $0<\mu<1$. Thus, the Universe can change the type of its equation of state from phantom energy to a more ordinary one, but only for such kind of the asymptote of the equation of state.

At $\mu>1$ we obtain $t_{0}=\infty$, i.e. the asymptotic approximation of $\rho+p=0$ condition. The evolution of such a Universe at the terminal stage practically coincides with the evolution of the flat Universe with a cosmological constant and without any other types of matter. There is no spacetime singularity in this case. Using the approximation (11) we obtain the asymptotes $\rho(t)-\rho_{0} \propto t^{1 /(1-\mu)}, \rho(t)+p(t) \propto t^{\mu /(1-\mu)} \rightarrow 0$ at $t \rightarrow \infty$. This solution can mimic the $\Lambda$ CDM model.

\subsubsection{Zero terminal density}

This last possibility assumes $\rho_{0}=H_{0}=0$, which means that a scale factor tends to some extremum. But this does not means an asymptotic expansion or contraction of the Universe is impossible. One simple example is the case $a \propto t^{\eta}, 0<\eta<1$ when the Universe keeps expanding, but $H$ decreases and tends to zero.

Let us consider the power-law asymptote of the equation of state

$$
\rho+p \underset{\rho \rightarrow 0}{\longrightarrow}-D \rho^{\nu}
$$

and substitute it into (6). The integral in (6) is finite at $\nu<1 / 2$, which yilds finite $t_{0}$. In this case $\rho \propto$ $\Delta t^{2 /(1-2 \nu)} \underset{t \rightarrow t_{0}}{\longrightarrow} 0, H \propto \Delta t^{1 /(1-2 \nu)} \underset{t \rightarrow t_{0}}{\longrightarrow} 0, \rho+p \propto$ $\Delta t^{2 \nu /(1-2 \nu)}$. If $0<\mu<1 / 2$, pressure tends to zero. This is a type IV singularity. If $\lambda=1+1 /(1-2 \nu)$ is a noninteger number, the higher derivatives of $H \propto$ $\Delta t^{\lambda-1}$ diverge. The condition $0<\mu<1 / 2$ means $\lambda>2$, so the first derivative of $H$ is finite, as well as both sides of the Friedmann equation (5). The value of 
Table 1: Possible cosmological singularities except "Big Bang" and "Big Crunch"

\begin{tabular}{|c|c|c|c|c|c|c|c|c|c|}
\hline $\mathrm{T}$ & Nickname & EoS & $\rho_{0}$ & $\left|p_{0}\right|$ & $p_{0}+\rho_{0}$ & & $\rho$ & $p+\rho$ & $a$ \\
\hline \multicolumn{10}{|c|}{$t \rightarrow t_{0}, \Delta t=t_{0}-t \rightarrow 0$} \\
\hline$\overline{\mathrm{I}}$ & "Big Rip" & $(1), w<-1$ & $\infty$ & $\infty$ & $-\infty$ & $\infty$ & $\propto \Delta t^{-2}$ & $\propto \Delta t^{-2}$ & $a \propto \Delta t^{-2 /(3|1+w|)}$ \\
\hline $\mathrm{I}$ & "Big Rip" & $(8), 1 / 2<\alpha<1$ & $\infty$ & $\infty$ & $-\infty$ & $\infty$ & $\propto \Delta t^{2 /(1-2 \alpha)}$ & $\propto \Delta t^{2 \alpha /(1-2 \alpha)}$ & $\ln a \propto \Delta t^{2(1-\alpha) /(1-2 \alpha)}$ \\
\hline III & "Big Freeze" & $(8), \alpha>1$ & $\infty$ & $\infty$ & $-\infty$ & $a_{0}$ & $\propto \Delta t^{2 /(1-2 \alpha)}$ & $\propto \Delta t^{2 \alpha /(1-2 \alpha)}$ & $\lambda=(2 \alpha-2) /(2 \alpha-1)$ \\
\hline II & "sudden" & (11), $\mu<0$ & $\rho_{0}$ & $\infty$ & $-\infty$ & $a_{0}$ & $\rho-\rho_{0} \propto \Delta t^{A}$ & $\propto \Delta t^{\mu A}$ & $a \rightarrow a_{0}-H_{0} \Delta t$ \\
\hline IV & & $(12), 0<\nu<1 / 2$ & 0 & 0 & 0 & $a_{0}$ & $\propto \Delta t^{2 /(1-2 \nu)}$ & $\propto \Delta t^{2 \nu /(1-2 \nu)}$ & $\lambda=(2-2 \nu) /(1-2 \nu)$ \\
\hline New & "Big Squeeze" & $(12), \nu<0$ & 0 & $\infty$ & $-\infty$ & $a_{0}$ & $\propto \Delta t^{2 /(1-2 \nu)}$ & $\propto \Delta t^{2 \nu /(1-2 \nu)}$ & $\lambda=(2-2 \nu) /(1-2 \nu)$ \\
\hline \multicolumn{10}{|c|}{$t \rightarrow \infty$} \\
\hline & "Little Rip" & $(8), 0<\alpha<1 / 2$ & $\infty$ & $\infty$ & $-\infty$ & $\infty$ & $\propto t^{2 /(1-2 \alpha)}$ & $\propto t^{2 \alpha /(1-2 \alpha)}$ & $\ln a \propto t^{2(1-\alpha) /(1-2 \alpha)}$ \\
\hline & "Little Rip" & $(8), \alpha<0$ & $\infty$ & $\infty$ & 0 & $\infty$ & $\propto t^{2 /(1-2 \alpha)}$ & $\propto t^{2 \alpha /(1-2 \alpha)}$ & $\ln a \propto t^{2(1-\alpha) /(1-2 \alpha)}$ \\
\hline & "Little Freeze" & $(12), 1 / 2<\nu<1$ & 0 & 0 & 0 & $a_{0}$ & $\propto t^{2 /(1-2 \nu)}$ & $\propto t^{2 \nu /(1-2 \nu)}$ & $a \rightarrow a_{0}-B t^{B}$ \\
\hline & "Little Freeze" & $(12), \nu>1$ & 0 & 0 & 0 & $\infty$ & $\propto t^{2 /(1-2 \nu)}$ & $\propto t^{2 \nu /(1-2 \nu)}$ & $\ln a \propto t^{2(\nu-1) /(2 \nu-1)}$ \\
\hline
\end{tabular}

$\lambda$ is the same as in (10). We can introduce the effective barotropic index $w=p / \rho \propto \Delta t^{(2 \nu-2) /(1-2 \nu)} \rightarrow \infty$.

If $\mu<0$ we have $|p| \underset{t \rightarrow t_{0}}{\longrightarrow} \infty$. This is a new type of the future singularity, which we name "Big Squeeze". It combines certain properties of the sudden singularity and the type IV singularity. It corresponds to $1<\lambda<$ 2 in (10). The first derivative of $H$ and both sides of the Friedmann equation (5) diverge. The asymptotics near this singularity type are $\rho \propto \Delta t^{2 /(1-2 \nu)} \underset{t \rightarrow t_{0}}{\longrightarrow} 0$, $H \propto \Delta t^{1 /(1-2 \nu)} \underset{t \rightarrow t_{0}}{\longrightarrow} 0,|p| \propto \Delta t^{2 \nu /(1-2 \nu)} \underset{t \rightarrow t_{0}}{\longrightarrow} \infty$, $a \underset{t \rightarrow t_{0}}{\longrightarrow} a_{0}+$ const $\Delta t^{(2-2 \nu) /(1-2 \nu)} \rightarrow a_{0}$. It requires the equation of state (12) with negative $\nu$. The example is the generalized Chaplygin gas which occurs in some cosmological theories.

At $1 / 2<\nu<1$ the integral in (6) diverges and $t_{0}=\infty$. In this case $\rho \propto t^{2 /(1-2 \nu)} \underset{t \rightarrow \infty}{\longrightarrow} 0, H \propto$ $t^{1 /(1-2 \nu)} \underset{t \rightarrow \infty}{\longrightarrow} 0, \rho+p \propto t^{2 \nu /(1-2 \nu)} \underset{t \rightarrow \infty}{\longrightarrow} 0, a \underset{t \rightarrow \infty}{\longrightarrow}$ $a_{0}-B t^{(2 \nu-2) /(2 \nu-1)}$. This is the mentioned above solution which could be named the "Little Freeze". In this case the effective barotropic index $w=p / \rho \propto$ $t^{(2 \nu-2) /(1-2 \nu)} \rightarrow \infty$.

At $\nu=1 / 2$ we can take into account the possible logarithmic factor and consider the asymptotic equation of state $\rho+p \underset{\rho \rightarrow 0}{\longrightarrow}-D \rho^{1 / 2}(\ln \rho)^{\beta}$. At $\beta>1$ we deal with the unconventional type IV singularity with $\ln \rho \propto \Delta t^{1 /(1-\beta)}$, at $\beta<1$ we deal with the "Little Freeze" with $\ln \rho \propto t^{1 /(1-\beta)}$. At $\beta=1$ we consider the equation of state with the asymptotic $\rho+p \underset{\rho \rightarrow 0}{\longrightarrow}-A \rho^{1 / 2}(\ln \rho)(\ln \ln \rho)^{\gamma}$, etc.

At $\nu>1$ we deal with the expanding Universe and $\ln a \propto t^{(2 \nu-2) /(2 \nu-1)} \underset{t \rightarrow \infty}{\longrightarrow} \infty$ at $D>0$ in spite of $H \propto t^{1 /(1-2 \nu)} \underset{t \rightarrow \infty}{\longrightarrow} 0$. This is the new "Little Freeze" case. The higher derivatives of $H$ diverge. At $\nu=1$ the Universe expands according to power law $a \propto t^{2 / 3 D}$. The effective barotropic index $w=p / \rho \rightarrow-1$.

\subsection{Open and closed models}

The second term in the right-hand side of (3) does not affects the properties of the singularities with $\rho, H \rightarrow \infty$ and $\rho \rightarrow \rho_{0} \neq 0, H \rightarrow H_{0} \neq 0$. The only exception is the "Big Crunch" singularity with $a \rightarrow 0$ which we do not study in this paper.

But we must revise a possibility of the existence and the properties of singularities with $H \rightarrow 0$ or $\rho \rightarrow 0$. A simple analysis shows that in all cases we have no new type of singularity. The equation of state (12) with $\nu<1$ could provide the type IV or the "Big Squeeze" singularities only for the flat model.

\section{Conclusion}

We tabulate all main cases of the cosmological singularities in Table $1 . \mathrm{T}$ and EoS mean type and equation of state, $\lambda$ corresponds to (10), $A=1 /(1-\mu)$, $B=2(1-\nu) /(1-2 \nu)$. The terminal values denoted $\rho_{0}$ and $a_{0}$ are finite and nonzero. Note that the "Big Squeeze" and the type IV cases are possible only for the flat Universe. The asymptote (11) of the equation of state at $0<\mu<1$ corresponds to changing the type of energy from phantom one to ordinary one or vice versa. At $\mu>1$ it provides an eternal near- $\Lambda \mathrm{CDM}$ expansion of the Universe.

Acknowledgements. Publications are based on the research provided by the grant support of the State Fund For Fundamental Research (projectF64/422015).

\section{References}

Caldwell R.R., Kamionkowski M., Weinberg N.N.: 2003, Phys. Rev. Lett., 91, 071301.

Nojiri S., Odintsov S.D., Tsujikawa S.: 2005, Phys. Rev. D, 71, 063004.

Frampton P.H., Ludwick K.J., Scherrer R.J.: 2011, Phys. Rev. D, 84, 083001. 